archives

of thermodynamics

Vol. 33(2012), No. 4, 91-108

DOI: $10.2478 / \mathrm{v} 10173-012-0029-5$

\title{
Evaluation of the thermodynamics of a four level system using canonical density matrix method
}

\author{
OLADUNJOYE A. AWOGA* \\ AKPAN A. IKOT \\ ENO E. ITUEN \\ LOUIS E. AKPABIO
}

Theoretical Physics Group, Department of Physics, University of Uyo, Nigeria

\begin{abstract}
We consider a four-level system with two subsystems coupled by weak interaction. The system is in thermal equilibrium. The thermodynamics of the system, namely internal energy, free energy, entropy and heat capacity, are evaluated using the canonical density matrix by two methods. First by Kronecker product method and later by treating the subsystems separately and then adding the evaluated thermodynamic properties of each subsystem. It is discovered that both methods yield the same result, the results obey the laws of thermodynamics and are the same as earlier obtained results. The results also show that each level of the subsystems introduces a new degree of freedom and increases the entropy of the entire system. We also found that the four-level system predicts a linear relationship between heat capacity and temperature at very low temperatures just as in metals. Our numerical results show the same trend.
\end{abstract}

Keywords: Canonical density matrix; Kronecker product, Reduced density matrix; Entropy

\section{Introduction}

The thermodynamics of a single system with four internal states have been calculated [1]. In quantum statistics the density matrix comes in handy

${ }^{*}$ Corresponding Author. E-mail address: ola.awoga@yahoo.com 
since it contains all the information about the system in consideration. Any information needed about the system can be deduced from the density matrix of the system. The density matrix thus naturally replaces the partition function when we seek to know thermodynamics of a quantum mechanical system. It has been established that both the density matrix method and partition function method yield the same results [2].

The thermodynamic properties of a system are guided by existing laws which include the laws of thermodynamics, the rule of Dulong and Petit for heat capacity of solids, Einstein model and Debye approximation for the heat capacity of solids, equipartition theorem and so on. The thermodynamics of any physical system should obey these laws.

The discussions of many literatures on thermodynamics and statistical mechanics [3-5] show that for a system in equilibrium with a heat bath in which only the energy is exchanged, the total value of any thermodynamic quantity for the entire system, i.e, the system and the reservoir, is equal to the sum of the values of that thermodynamic quantity for each the subsystem. Such a system is called a canonical ensemble.

In this work our aim is to calculate the thermodynamics of a four-level system with two subsystems via the canonical density matrix method and show that our results are in concordance with existing laws. We also aim to show that the four level system can be used to predict the heat capacity of metals at very low temperatures.

The paper is organized as follows. In Section 2, we take a cursory look at the mathematical background for tensor product of matrices. In Section 3, we present a brief review of the density matrix. In Section 4, we highlight the thermodynamics properties we wish to calculate. In Sections 5 and 6 , we calculate the thermodynamic properties of the system using the tensor product method, and treating the subsystems separately using the reduced densities of each subsystems respectively. In Section 7 we give a brief conclusion.

\section{Mathematical background}

Numerous textbooks and papers of mathematics have discussed in details the algebra of matrices and tensor algebra of matrices [6-11]. The tensor product of matrices commonly known as the Kronecker product is different from the normal row $\times$ column product. In mathematics, the Kronecker product, denoted by $\otimes$, is an operation on two matrices of arbitrary size 
resulting in a block matrix. It gives the tensor product with respect to a standard choice of basis [12]. The following definitions give an insight into Kronecker product and Kronecker sum.

Let $A \in \Re^{m \times n}, B \in \Re^{p \times q}$, then the Kronecker product of matrices $A$ and $B$ is defined as the matrix

$$
A \otimes B=\left[\begin{array}{lll}
a_{11} B & \cdots & a_{1 n} B \\
\vdots & \ddots & \\
a_{m 1} B & & a_{m n} B
\end{array}\right] \in \Re^{m p \times n q},
$$

where $\Re$ is the set of real numbers. Equation (1) holds for both real and complex valued matrices $A$ and $B$. For example if

$$
A=\left(\begin{array}{ll}
a_{11} & a_{12} \\
a_{21} & a_{22}
\end{array}\right) \quad \text { and } \quad B=\left(\begin{array}{ll}
b_{11} & b_{12} \\
b_{21} & b_{22}
\end{array}\right)
$$

then

$$
A \otimes B=\left(\begin{array}{ll}
a_{11}\left[\begin{array}{ll}
b_{11} & b_{12} \\
b_{21} & b_{22}
\end{array}\right] & a_{11}\left[\begin{array}{ll}
b_{11} & b_{12} \\
b_{21} & b_{22}
\end{array}\right] \\
a_{21}\left[\begin{array}{ll}
b_{11} & b_{12} \\
b_{21} & b_{22}
\end{array}\right] & a_{22}\left[\begin{array}{ll}
b_{11} & b_{12} \\
b_{21} & b_{22}
\end{array}\right]
\end{array}\right),
$$

therefore

$$
A \otimes B=\left(\begin{array}{cccc}
a_{11} b_{11} & a_{11} b_{12} & a_{12} b_{11} & a_{12} b_{12} \\
a_{11} b_{21} & a_{11} b_{22} & a_{12} b_{21} & a_{12} b_{22} \\
a_{21} b_{11} & a_{21} b_{12} & a_{22} b_{11} & a_{22} b_{12} \\
a_{21} b_{21} & a_{21} b_{22} & a_{22} b_{21} & a_{22} b_{22}
\end{array}\right) .
$$

It is clear that

$$
A \otimes B \neq B \otimes A .
$$

For any

$$
B \in \Re^{p \times q}, \quad I_{2} \otimes B=\left[\begin{array}{ll}
B & 0 \\
0 & B
\end{array}\right],
$$

where $I_{2}$ is the $2 \times 2$ identity matrix. Replacing $I_{2}$ by $I_{n}$ yields a block diagonal matrix with $n$ copies of $B$ along the diagonal.

We list some properties of the Kronecker product

(i) Let $A \in \Re^{m \times n}, B \in \Re^{r \times s}, C \in \Re^{n \times p}$ and $D \in \Re^{s \times t}$, then

$$
(A \otimes B)(C \otimes D)=A C \otimes B D\left(\in \Re^{m r \times p t}\right) .
$$

(ii) For all $A$ and $B$,

$$
(A \otimes B)^{T}=A^{T} \otimes B^{T} \quad,
$$


where superscript $T$ denotes the matrix transpose.

(iii) If $A \in \Re^{n \times n}$ and $B \in \Re^{m \times m}$ are symmetric, then $A \otimes B$ is symmetric.

(iv) If $A$ and $B$ and nonsingular, then

$$
(A \otimes B)^{-1}=A^{-1} \otimes B^{-1} .
$$

(v) Let $\mathrm{A} \in \Re^{n \times n}$ and $\mathrm{B} \in \Re^{m \times m}$. Then the Kronecker sum or tensor sum of $A$ and $B$ denoted $A \oplus B$, is the $m n \times m n$ matrix

$$
A \oplus B=\left(I_{m} \otimes A\right)+\left(B \otimes I_{n},\right)
$$

where $I$ represents the identity matrix of the appropriate dimension. In general,

$$
A \oplus B \neq B \oplus A .
$$

(vi) Let $A \in \Re^{n \times n}$ have eigenvalues $\lambda_{i}, i \in \underline{n}$ and let $B \in \Re^{m \times m}$ have eigenvalues $N_{j}, j \in m$, then the Kronecker sum $A \oplus B$ has $m n$ eigenvalues

$$
\lambda_{1}+\mu_{1}, \ldots \lambda_{1}+\mu_{m}, \lambda_{2}+\mu_{1}, \ldots, \lambda_{2}+\mu_{m}, \ldots, \lambda_{n}+\mu_{m} \ldots
$$

The Kronecker sum has an important property given by the identity

$$
e^{A} \otimes e^{B}=e^{A \oplus B} .
$$

Let $A \in \Re^{m \times n}$ and $B \in \Re^{p \times q}$, the partial trace $\operatorname{Tr}_{A}$ is a linear map from $\Re^{m p \times n q}$ to $\Re^{n \times m}$ that is determined by the equation

$$
\operatorname{Tr}_{B}(A \otimes B)=\operatorname{Tr}(B) A
$$

for all $A \in \Re^{n \times m}$ and $B \in \Re^{p \times q}$, extending to all of $\Re^{m p \times n q}$ by linearity. Similarly $\operatorname{Tr}_{B}: \Re^{m p \times n q} \rightarrow \Re^{p \times q}$

$$
\operatorname{Tr}_{A}(A \otimes B)=\operatorname{Tr}(A) B .
$$

\section{Review of density matrix}

The density matrix is a positive semidefinite matrix of trace one. Detailed discussions on density matrix is available in literature [13-24]. Here we present a brief discussion of the density matrix.

Consider an ensemble of systems with a total number of $z$ members or subsystems and each having a state vector $\left|\psi^{(\alpha)}\right\rangle, \alpha=1,2,3, \ldots ., z$ [25]. 
Suppose each state vector of each member of the ensemble can be expanded in a sum

$$
\left|\psi^{(\alpha)}\right\rangle=\sum_{k} C_{k}^{(\alpha)}\left|\phi_{k}\right\rangle
$$

where the $\left|\phi_{k}\right\rangle$ are orthonormal sets with property

$$
\left\langle\phi_{i} \mid \phi_{j}\right\rangle=\delta_{i j}
$$

and the coefficients $C_{k}^{(\alpha)}$ are components.

The expectation value of an observable $A$ averaged over the ensemble of systems is given by

$$
\langle A\rangle=\frac{1}{z} \sum_{\alpha=1}^{z}\left\langle\psi^{(\alpha)}|\hat{A}| \psi^{(\alpha)}\right\rangle
$$

which in this cases gives

$$
\langle A\rangle=\sum_{k, l}\left(\frac{1}{z} \sum_{\alpha=1} C_{k}^{(\alpha) *} C_{l}^{(\alpha)}\right)\left\langle\phi_{k}|\hat{A}| \phi_{l}\right\rangle,
$$

where the symbols ${ }^{\wedge}$ and $*$ denote operator and complex conjugate respectively.

Defining a matrix

$$
\hat{\rho}_{l k}=\sum_{\alpha=1}^{z} C_{l}^{(\alpha)} C_{k}^{(\alpha) *}
$$

thus its average is

$$
\bar{\rho}_{l k}=\frac{1}{z} \sum_{\alpha=1}^{z} C_{l}^{(\alpha)} C_{k}^{(\alpha) *}
$$

and let the elements of

$$
A_{k l}=\left\langle\phi_{k}|\hat{A}| \phi_{l}\right\rangle
$$

be the elements of matrix $A$ therefore using Eqs. (15) and (17) in Eq. (14) we have

$$
\langle A\rangle=\frac{1}{z} \sum_{k, l} \hat{\rho}_{l k} A_{k l} \quad=\frac{1}{z} \operatorname{Tr}(\hat{\rho} A)=\operatorname{Tr}(\bar{\rho} A),
$$

where $\hat{\rho}$ and $A$ are matrices with elements $\rho_{l k}$ and $A_{k l}$ in the basis of vectors $\left\{\left|\phi_{k}\right\rangle\right\}$. The matrix $\hat{\rho}_{l k}$ is called the density matrix operator and $\bar{\rho}$ is the density matrix. Tr is the trace operator. 
For a canonical ensemble, the density matrix takes the form

$$
\rho=e^{-\beta H},
$$

where $H$ is the Hamiltonian of the system with $k_{B}$ the Boltzmann constant and $T$ absolute temperature. (The subscript $B$ has nothing to do with system B).

Now consider two quantum systems, A and B, with wavevectors $\left|\psi_{A}^{(\alpha)}\right\rangle$ and $\left|\psi_{B}^{(\alpha)}\right\rangle$ each in a Hilbert space $H_{A}$ and $H_{B}$ respectively. Let $\left|\psi_{A B}^{(\alpha)}\right\rangle$ be the wavevector of this bipartite composite system. The composite systems is in the Hilbert space $H_{A} \otimes H_{B}$ i.e. $\left|\psi_{A B}^{(\alpha)}\right\rangle \in H_{A} \otimes H_{B}$.

Let $\rho_{A}$ and $\rho_{B}, H_{A}$ and $H_{B}$ be the density matrices and Hamiltonians of the systems. The density matrix of the combined system is given by [26]

$$
\rho=\rho_{A} \otimes \rho_{B}
$$

and the total Hamiltonian is given by

$$
H=H_{A} \oplus H_{B}
$$

The density matrix of the subsystems $\rho_{A}$ can be retrieved from the density matrix of entire system $\rho$. To do this, we take the partial trace over system B. Using Eq. (11) and Eq. (21)

$$
\rho_{A}=\operatorname{Tr}_{B}(\rho)
$$

similarly

$$
\rho_{B}=\operatorname{Tr}_{A}(\rho),
$$

where $\rho_{A}$ and $\rho_{B}$ are called the reduced density matrix of subsystems A and $B$ respectively. $\operatorname{Tr}_{B}$ and $\operatorname{Tr}_{A}$ are the partial trace over $\mathrm{B}$ and partial trace over A respectively. Equations (24) and (25) are possible because of the property that the trace of the density matrix is unity.

\section{Thermodynamic properties}

Profound details on thermodynamic properties can be found in many textbooks [27-28]. We highlight here some thermodynamics, relevant to a canonical ensemble 
(i) Internal energy or average energy $\langle E\rangle$ : It is the total energy of the system. It is the expectation value or ensemble average for the energy. It is related to the density matrix by

$$
\langle E\rangle=\operatorname{Tr}(\bar{\rho} H) .
$$

If a system has two subsystems $A$ and $B$ each with internal energies $\left\langle E_{A}\right\rangle$ and $\left\langle E_{B}\right\rangle$ then the total internal energy of the combined system is

$$
\langle E\rangle=\left\langle E_{A}\right\rangle+\left\langle E_{B}\right\rangle .
$$

(ii) Helmholtz free energy $(F)$ : This is a thermodynamic potential which measures the useful work obtainable from a closed thermodynamic system at a constant temperature and volume. For such a system, the negative of the Helmholtz energy gives the maximum amount of work extractable from a canonical ensemble. In terms of the density matrix it is given by

$$
F=-\frac{1}{\beta} \ln [\operatorname{Tr}(\rho)]
$$

or

$$
F=\operatorname{Tr}(\bar{\rho} H)-\operatorname{Tr}(\bar{\rho} S) T .
$$

Similarly for a system with subsystems A and B

$$
F=F_{A}+F_{B} .
$$

(iii) Entropy: It measures the degree of disorder in a system, measures our ignorance about a system and measures the irreversible changes in a system. It is given by

$$
S=-k_{B} \operatorname{Tr}[\bar{\rho} \ln \bar{\rho}],
$$

and also for a system with subsystems A and B

$$
S=S_{A}+S_{B},
$$

(iv) Specific heat capacity: It is the amount of energy needed to raise the temperature of one kilogram of a substance by one Kelvin. It depends on the temperature of the system. The heat capacity at constant pressure $C_{p}$ is useful to chemist whereas it is the capacity at constant volume, $C_{v}$ that is important to physicists. It is given by

$$
C_{v}=\frac{\partial\langle E\rangle}{\partial T}=\frac{-1}{k_{B} T^{2}} \frac{\partial}{\partial \beta}[\operatorname{Tr}(\bar{\rho} H)] .
$$




\section{$5 \quad$ Kronecker product method}

Consider a four-level system consisting of two subsystems A and B. The subsystems are described by a simple particle Hamiltonians of the form

$$
H_{A}=\left(\begin{array}{llll}
\epsilon_{0} & 0 & 0 & 0 \\
0 & \epsilon_{0} & 0 & 0 \\
0 & 0 & \epsilon_{0} & 0 \\
0 & 0 & 0 & \epsilon_{0}
\end{array}\right)
$$

and

$$
H_{B}=\left(\begin{array}{llll}
-\epsilon_{1} & 0 & 0 & 0 \\
0 & \epsilon_{1} & 0 & 0 \\
0 & 0 & \epsilon_{1} & 0 \\
0 & 0 & 0 & -\epsilon_{1}
\end{array}\right),
$$

where $\epsilon_{0}$ is the energy of the ground state which is constant and $\epsilon_{1}$ is the energy of an agitated state. The system is in thermal equilibrium with a reservoir at temperature $T$. Using the formula for the density matrix of a canonical ensemble Eq. (21) we obtain the density matrix of each of the subsystems as

$$
\rho_{A}=\left(\begin{array}{llll}
e^{-\beta \epsilon_{0}} & 0 & 0 & 0 \\
0 & e^{-\beta \epsilon_{0}} & 0 & 0 \\
0 & 0 & e^{-\beta \epsilon_{0}} & 0 \\
0 & 0 & 0 & e^{-\beta \epsilon_{0}}
\end{array}\right)
$$

and

$$
\rho_{B}=\left(\begin{array}{llll}
e^{\beta \epsilon_{1}} & 0 & 0 & 0 \\
0 & e^{-\beta \epsilon_{1}} & 0 & 0 \\
0 & 0 & e^{-\beta \epsilon_{1}} & 0 \\
0 & 0 & 0 & e^{\beta \epsilon_{1}}
\end{array}\right)
$$

Now the total Hamiltonian of the entire system is

$$
H=H_{A} \oplus H_{B}=\left(I_{4 \times 4} \otimes H_{A}\right)+\left(H_{B} \otimes I_{4 \times 4}\right)
$$

where

$$
I_{4 \times 4}=\left(\begin{array}{cccc}
1 & 0 & 0 & 0 \\
0 & 1 & 0 & 0 \\
0 & 0 & 1 & 0 \\
0 & 0 & 0 & 1
\end{array}\right)
$$


Using Eq. (1), we have the Hamiltonian of the entire system as the $16 \times 16$ matrix, which we have written in block form as a $4 \times 4$ block matrix

$$
H=\left[\begin{array}{llll}
\left(\epsilon_{1}-\epsilon_{0}\right) I_{4 \times 4} & 0 I_{4 \times 4} & 0 I_{4 \times 4} & 0 I_{4 \times 4} \\
0 I_{4 \times 4} & \left(\epsilon_{1}+\epsilon_{0}\right) I_{4 \times 4} & 0 I_{4 \times 4} & 0 I_{4 \times 4} \\
0 I_{4 \times 4} & 0 I_{4 \times 4} & \left(\epsilon_{1}+\epsilon_{0}\right) I_{4 \times 4} & 0 I_{4 \times 4} \\
0 I_{4 \times 4} & 0 I_{4 \times 4} & 0 I_{4 \times 4} & \left(\epsilon_{1}-\epsilon_{0}\right) I_{4 \times 4}
\end{array}\right],
$$

The joint density matrix is obtained by using the identity in Eq. (10). It is also a $16 \times 16$ matrix

$$
\rho=\left[\begin{array}{llll}
e^{-\beta\left(\epsilon_{1}-\epsilon_{0}\right)} I_{4 \times 4} & 0 I_{4 \times 4} & 0 I_{4 \times 4} & 0 I_{4 \times 4} \\
0 I_{4 \times 4} & e^{-\beta\left(\epsilon_{1}+\epsilon_{0}\right)} I_{4 \times 4} & 0 I_{4 \times 4} & 0 I_{4 \times 4} \\
0 I_{4 \times 4} & 0 I_{4 \times 4} & e^{-\beta\left(\epsilon_{1}+\epsilon_{0}\right)} I_{4 \times 4} & 0 I_{4 \times 4} \\
0 I_{4 \times 4} & 0 I_{4 \times 4} & 0 I_{4 \times 4} & e^{-\beta\left(\epsilon_{1}-\epsilon_{0}\right)} I_{4 \times 4}
\end{array}\right]
$$

Normalizing the density matrix, we obtain the average density matrix as

$$
\bar{\rho}=\frac{1}{16 \cosh \left(\beta \epsilon_{1}\right)}\left[\begin{array}{llll}
e^{\beta \epsilon_{1}} I_{4 \times 4} & 0 I_{4 \times 4} & 0 I_{4 \times 4} & 0 I_{4 \times 4} \\
0 I_{4 \times 4} & e^{-\beta \epsilon_{1}} I_{4 \times 4} & 0 I_{4 \times 4} & 0 I_{4 \times 4} \\
0 I_{4 \times 4} & 0 I_{4 \times 4} & e^{-\beta \epsilon_{1}} I_{4 \times 4} & 0 I_{4 \times 4} \\
0 I_{4 \times 4} & 0 I_{4 \times 4} & 0 I_{4 \times 4} & e^{\beta \epsilon_{1}} I_{4 \times 4}
\end{array}\right] .
$$

The matrices in Eqs. (39)-(42) are all $16 \times 16$ matrices however, we have written them in a block form. The $16 \times 16$ matrices can be recovered by making use of Eqs. (3) and (4).

Using Eq. (26), we evaluate the internal energy of the system as

$$
\langle E\rangle=\frac{1}{2 \cosh \left(\beta \epsilon_{1}\right)}\left[\left(\epsilon_{0}+\epsilon_{1}\right) e^{-\beta \epsilon_{1}}+\left(\epsilon_{0}-\epsilon_{1}\right) e^{\beta \epsilon_{1}}\right]=\epsilon_{0}-\epsilon_{1} \tanh \left(\beta \epsilon_{1}\right) .
$$

This is the same result as obtained in [1]. The changes in this energy with temperature are shown in Figs. 1 and 2 for positive and negative values of $\epsilon_{1}$, respectively. The energy increases with temperature and has a maximum value of $\epsilon_{0}$ irrespective of the sign of $\epsilon_{1}$.

We obtain the Helmholtz free energy from Eq. (28) as

$$
F=-\frac{1}{\beta} \ln \left[16 e^{-\beta \epsilon_{0}} \cosh \left(\beta \epsilon_{1}\right)\right]=\epsilon_{0}-\frac{1}{\beta} \ln \left[16 \cosh \left(\beta \epsilon_{1}\right)\right] .
$$

Equation (43) has the same form as that one obtained in [1]. The changes of the Helmholtz free energy with temperature is depicted in Fig. 3. It 


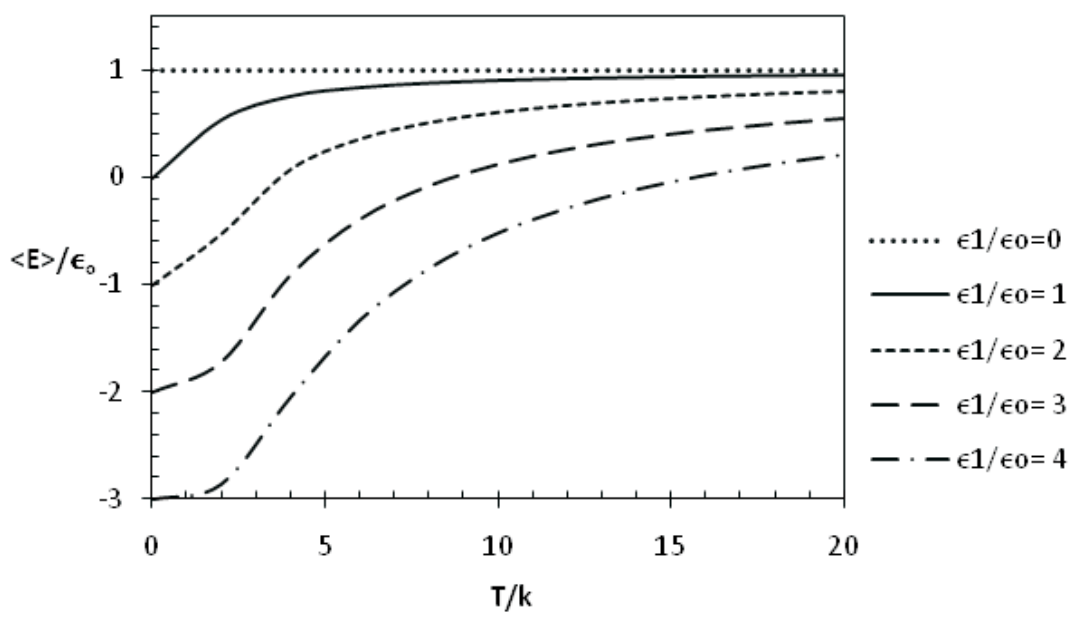

Figure 1. Internal energy $\langle E\rangle / \epsilon_{0}$ versus temperature $T$ for different positive values of $\epsilon_{1}$.

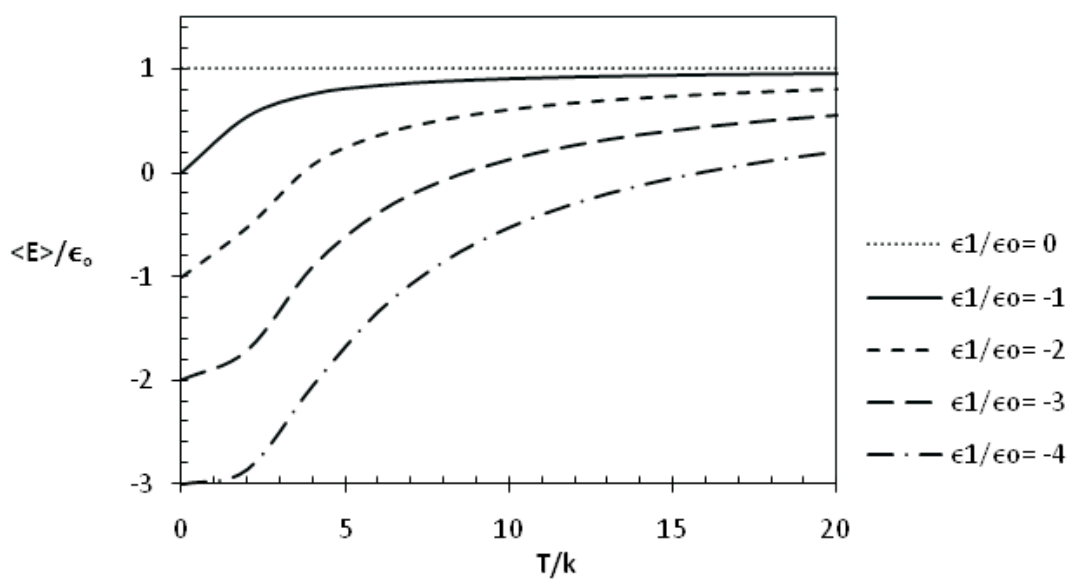

Figure 2. Internal energy $\langle E\rangle / \epsilon_{0}$ versus temperature $T$ for different negative values of $\epsilon_{1}$.

increases negatively and indefinitely with temperature, hence it meets up with the expectations of thermodynamics. Like the internal energy, it is not affected by the sign of $\epsilon_{1}$. 


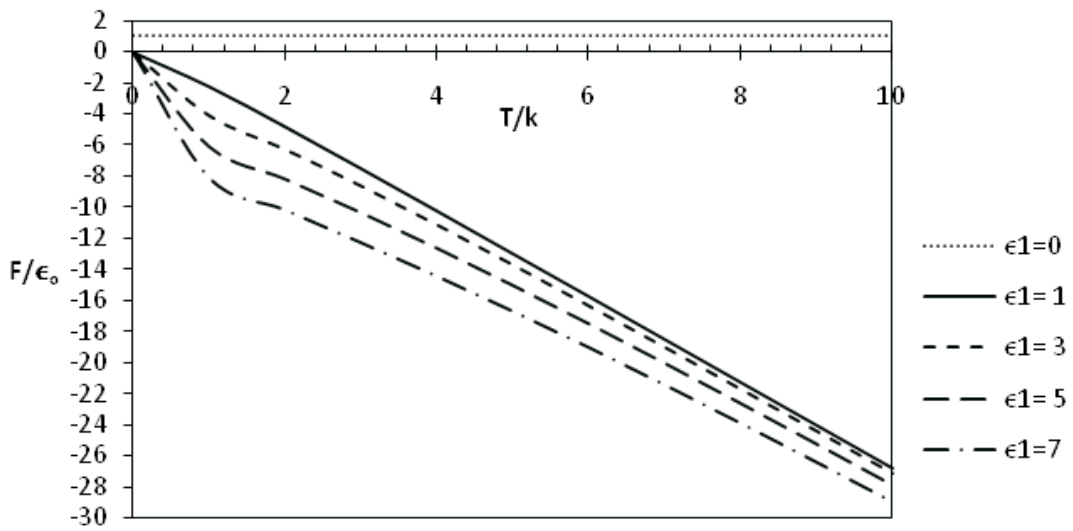

Figure 3. Free energy versus temperature $T$ for different values of $\epsilon_{1}$.

From Eq. (31), the entropy is calculated as

$$
\begin{aligned}
S & =-\frac{k_{B}}{2 \cosh \left(\beta \epsilon_{1}\right)}\left[\left(\beta \epsilon_{1}-\ln \left[16 \cosh \beta \epsilon_{1}\right]\right) e^{\beta \epsilon_{1}}\left(\beta \epsilon_{1}+\ln \left[16 \cos \left(\beta \epsilon_{1}\right)\right]\right) e^{-\beta \epsilon_{1}}\right] \\
& =k_{B} \ln \left[16 \cosh \beta \epsilon_{1}\right]-\frac{\epsilon_{1}}{T} \tanh \left(\beta \epsilon_{1}\right) .
\end{aligned}
$$

The entropy also has the same form as that obtained in [1]. Like the free energy it has increased by $k_{B} \ln 4$. The entropy fulfills both the second and third laws of thermodynamics viz $\Delta S \geq 0$ and $T \rightarrow 0, S \rightarrow 0$ respectively. The entropy changes with temperature are shown in Fig. 4 . It has a maximum value of approximately $2.773 k_{B}$.

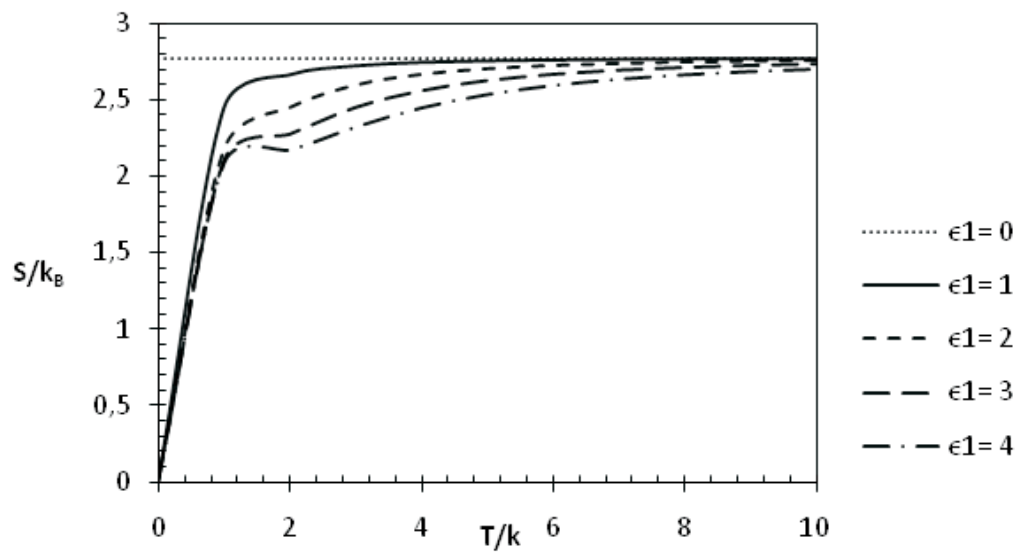

Figure 4. Entropy $S$ versus temperature $T$ for different values of $\epsilon_{1}$. 
Finally, the specific heat capacity is evaluated as

$$
C_{v}=-\frac{1}{k_{B} T^{2}} \frac{\partial}{\partial \beta}\left[\epsilon_{0}-\epsilon_{1} \tan \beta \epsilon_{1}\right]=k_{B}\left(\frac{\epsilon_{1}}{k_{B} T}\right)^{2} \operatorname{sech}^{2}\left(\beta \epsilon_{1}\right)
$$

The plot of Eq. (45) is as displayed in Fig. 5. As $\epsilon_{1}$ increases $C_{v}$ increases and it is independent of the sign of $\epsilon_{1}$. It also goes to zero as $T \rightarrow 0$ as predicted by thermodynamics. The $C_{v}$ can be written as an infinite series

$$
C_{v}=k_{B}\left(\frac{\epsilon_{1}}{k_{B} T}\right)^{2} \sum_{n=0}^{\infty}\left[(2 n+1) e^{-2(2 n+1) \beta \epsilon_{1}}-2 n e^{-4 n \beta \epsilon_{1}}\right]
$$

The typical plots of the heat capacity versus temperature are shown in Fig. 6 and 7 for the first few values of $n$ for different values of $\epsilon_{1}$. It could be noticed that $C_{v}$ increases with increasing $\epsilon_{1}$.

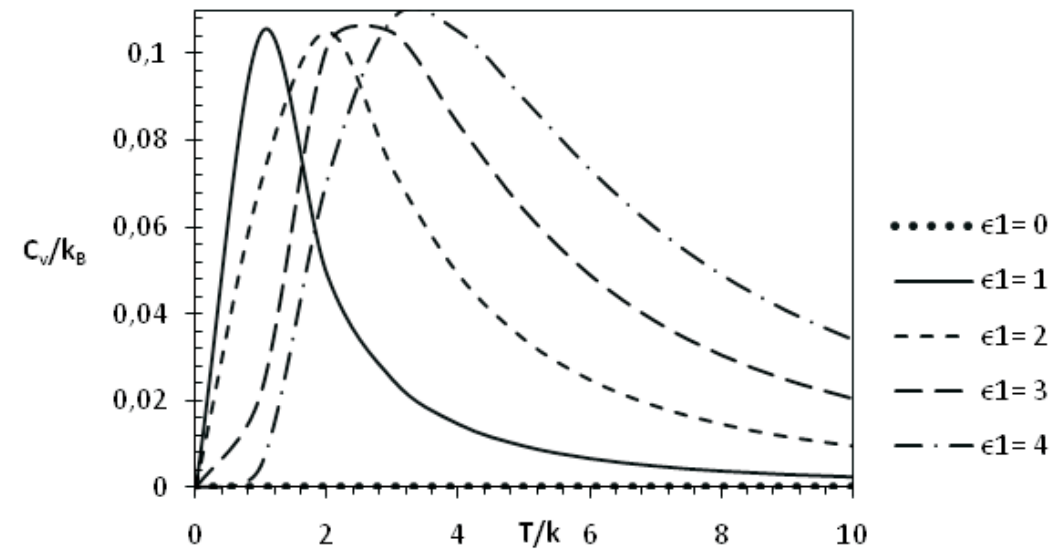

Figure 5. Heat capacity $C_{v}$ versus temperature $T$ for different values of $\epsilon_{1}$.

\section{Treating the subsystems separately}

We now treat each subsystem separately and evaluate their thermodynamics individually. The density matrix of each subsystem is the reduced density matrix which can be obtained from the joint density matrix $\rho$ in Eq. (40) by partial trace method. Using Eqs. (11) and Eq. (12) we obtain density 


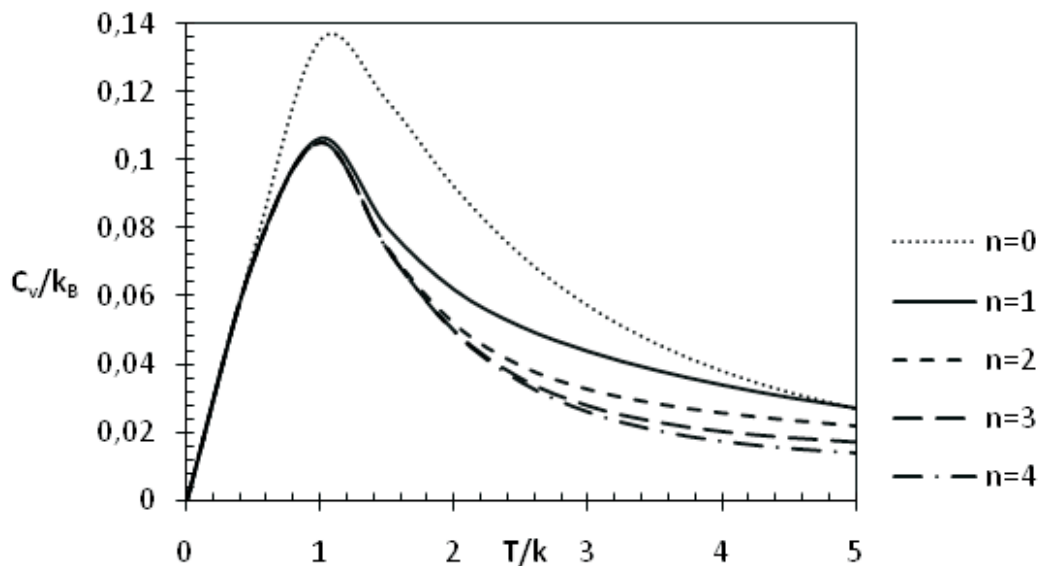

Figure 6. Heat capacity $C_{v}$ versus temperature $T$ for different values of $n$ with $\epsilon_{1}=1$.

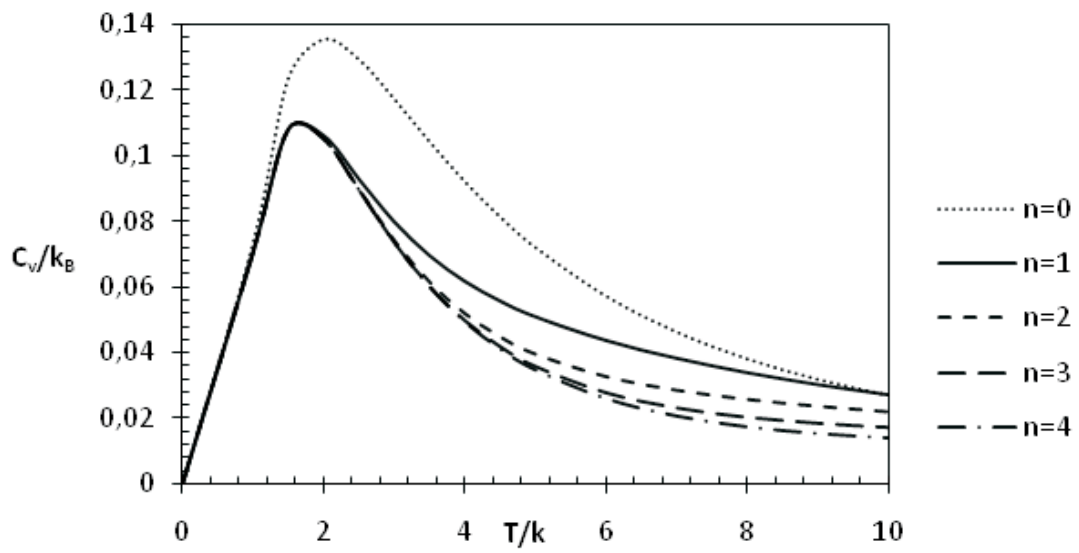

Figure 7. Heat capacity $C_{v}$ versus temperature $T$ for different values of $n$ with $\epsilon_{1}=2$.

matrices as

$$
\begin{gathered}
\hat{\rho}_{A}=\left(\begin{array}{llll}
e^{-\beta \epsilon_{0}} & 0 & 0 & 0 \\
0 & e^{-\beta \epsilon_{0}} & 0 & 0 \\
0 & 0 & e^{-\beta \epsilon_{0}} & 0 \\
0 & 0 & 0 & e^{-\beta \epsilon_{0}}
\end{array}\right), \\
\hat{\rho}_{B}=\left(\begin{array}{llll}
e^{\beta \epsilon_{1}} & 0 & 0 & 0 \\
0 & e^{-\beta \epsilon_{1}} & 0 & 0 \\
0 & 0 & e^{-\beta \epsilon_{1}} & 0 \\
0 & 0 & 0 & e^{\beta \epsilon_{1}}
\end{array}\right),
\end{gathered}
$$


and normalizing them

$$
\begin{gathered}
\bar{\rho}_{A}=\frac{1}{4}\left(\begin{array}{cccc}
1 & 0 & 0 & 0 \\
0 & 1 & 0 & 0 \\
0 & 0 & 1 & 0 \\
0 & 0 & 0 & 1
\end{array}\right), \\
\bar{\rho}_{B}=\frac{1}{4 \cosh \left(\beta \epsilon_{1}\right)}\left(\begin{array}{llll}
e^{\beta \epsilon_{1}} & 0 & 0 & 0 \\
0 & e^{-\beta \epsilon_{1}} & 0 & 0 \\
0 & 0 & e^{-\beta \epsilon_{1}} & 0 \\
0 & 0 & 0 & e^{\beta \epsilon_{1}}
\end{array}\right) .
\end{gathered}
$$

The internal energy of subsystem A is

$$
\left\langle E_{A}\right\rangle=\operatorname{Tr}\left(\bar{\rho}_{A} H_{A}\right)=\epsilon_{0},
$$

and the internal energy of $\mathrm{B}$ is

$$
\left\langle E_{B}\right\rangle=\operatorname{Tr}\left(\bar{\rho}_{B} H_{B}\right)=\frac{1}{2 \cosh \left(\beta \epsilon_{1}\right)}\left[\epsilon_{1} e^{-\beta \epsilon_{1}}-\epsilon_{1} e^{\beta \epsilon_{1}}\right]=-\epsilon_{1} \tanh \left(\beta \epsilon_{1}\right)
$$

therefore the total internal energy of system is

$$
\langle E\rangle=\left\langle E_{A}\right\rangle+\left\langle E_{B}\right\rangle=\epsilon_{0}-\epsilon_{1} \tanh \left(\beta \epsilon_{1}\right) .
$$

The free energy of subsystem $\mathrm{A}$ is

$$
F_{A}=-\frac{1}{\beta} \ln \left[\operatorname{Tr}\left(\hat{\rho}_{A}\right)\right]=-\frac{1}{\beta} \ln \left[4 e^{-\beta \epsilon_{0}}\right]=\epsilon_{0}-\frac{\ln 4}{\beta},
$$

while the free energy of $B$ is

$$
F_{B}=-\frac{1}{\beta} \ln \left[\operatorname{Tr}\left(\hat{\rho}_{B}\right)\right]=-\frac{1}{\beta} \ln \left[4 \cosh \beta \epsilon_{1}\right],
$$

thus the total free energy of the system is

$$
F=F_{A}+F_{B}=\epsilon_{0}-\frac{\ln 4}{\beta}-\frac{1}{\beta} \ln \left[4 \cosh \left(\beta \epsilon_{1}\right)\right] .
$$

For entropy of subsystem A and B we respectively get

$$
S_{A}=k_{B} \ln 4
$$




$$
\begin{aligned}
S_{B} & =\frac{-k_{B}}{4 \cosh \left(\beta \epsilon_{1}\right)}\left[\beta \epsilon_{1}\left(e^{\beta \epsilon_{1}}-e^{-\beta \epsilon_{1}}-\ln \left[4 \cosh \left(\beta \epsilon_{1}\right)\right] e^{\beta \epsilon_{1}}+e^{-\beta \epsilon_{1}}\right)\right] \\
& =k_{B} \ln \left[4 \cosh \left(\beta \epsilon_{1}\right)\right]-\frac{\epsilon_{1}}{T} \tanh \beta \epsilon_{1}
\end{aligned}
$$

and therefore

$$
\begin{aligned}
& S=S_{A}+S_{B}=k_{B} \ln 4+k_{B} \ln \left[4 \cosh \left(\beta \epsilon_{1}\right)\right]-\frac{\epsilon_{1}}{T} \tanh \left(\beta \epsilon_{1}\right) \\
& =k_{B} \ln \left[16 \cosh \left(\beta \epsilon_{1}\right)\right]-\frac{\epsilon_{1}}{T} \tanh \left(\beta \epsilon_{1}\right) .
\end{aligned}
$$

For heat capacity, using (32), we obtain

$$
\begin{gathered}
C_{v A}=0 \\
C_{v B}=k_{B}\left(\frac{\epsilon_{1}}{k_{B} T}\right)^{2} \operatorname{sech}^{2}\left(\beta \epsilon_{1}\right), \\
C_{v}=C_{v B}+C_{v A}=k_{B}\left(\frac{\epsilon_{1}}{k_{B} T}\right)^{2} \operatorname{sech}^{2}\left(\beta \epsilon_{1}\right) .
\end{gathered}
$$

The heat capacity is independent of $\epsilon_{0}$, this is expected since $\epsilon_{0}$ is the ground state energy and according to thermodynamics $C_{v} \rightarrow 0$ at ground state.

The results, Eqs. (53), (56), (59) and (62), are the same as the corresponding results of Eqs. (42), (43), (44) and (45) for the Kronecker product method. This proofs the equivalence of the methods.

We can write the heat capacity in exponential forms

$$
C_{v}=4 k_{B}\left(\frac{\epsilon_{1}}{k_{B} T}\right)^{2} \frac{e^{2 \beta \epsilon_{1}}}{\left(e^{2 \beta \epsilon_{1}}+1\right)^{2}}=\epsilon_{1} \frac{d f\left(\epsilon_{1}\right)}{d T}
$$

where $f\left(\epsilon_{1}\right)=\frac{1}{e^{2 \beta \epsilon_{1}}+1}$ is the Fermi-Dirac statistics. This means that the particles in the four-level system are fermions and therefore obey the FermiDirac statistics. At very low temperatures the heat capacity in Eq. (63) can be written as [29]

$$
C_{v}=\int_{0}^{\infty} d \epsilon_{1}\left(\epsilon_{1}-\epsilon_{0}\right) \frac{d f\left(\epsilon_{1}\right)}{d T} D\left(\epsilon_{1}\right)
$$

where $D\left(\epsilon_{1}\right)$ is the density of states of the system.

For very low temperatures $k_{B} T<\epsilon_{0}, \frac{d f}{d T}$ is only large when $\epsilon_{1}$ is close to $\epsilon_{0}$ [29]. Thus it is good to evaluate the density of states at $\epsilon_{0}$ so that 
$D\left(\epsilon_{1}\right)=D\left(\epsilon_{0}\right)$ at low temperatures. Hence from Eq. (64), the heat capacity of the four-level system at low temperatures is

$$
C_{v}=k_{B}^{2} T D\left(\epsilon_{0}\right) \int_{0}^{\infty} \frac{x^{2} e^{x}}{\left(e^{x}+1\right)^{2}} d x,
$$

where $x=\beta\left(\epsilon_{1}-\epsilon_{0}\right)$. The lower limit is chosen as $-\infty$ because $e^{x}$ is already negligible at $-\beta \epsilon_{0}$ when $\epsilon_{1}=0$. Integral in Eq. (65) is a standard integral and its value equal to $\pi^{2} / 3$ can be obtained from standard tables $[29,30]$. Hence the heat capacity at low temperature is

$$
C_{v}=\frac{1}{3} \pi^{2} k_{B}^{2} T D\left(\epsilon_{0}\right) .
$$

If the system contains $N$ free electrons in three-dimensions [29], then

$$
D\left(\epsilon_{0}\right)=\frac{3 N}{2 \epsilon_{0}},
$$

hence, the heat capacity becomes

$$
C_{v}=\frac{\pi^{2} k_{B}^{2}}{2 \epsilon_{0}} T .
$$

If we set $\epsilon_{0}=\epsilon_{F}$, where $\epsilon_{F}$ is the Fermi energy, we get the same result as Eq. (16) in [29] (page 136).

Equations (66) and (68) predict a linear relationship between heat capacity of the four-level system and temperature at very low temperature. This is known to be so for metals. This result is also given by the free gas electron model. This linear relationship between $C_{v}$ and $T$ at low temperatures can be seen in Figs. 6 and 7 between $0 \leq T \leq 1$. Thus, the four-level can be used to model the heat capacity of metals for electronic contribution along with the Debye approximation for phonon contribution.

\section{Conclusion}

We have evaluated the thermodynamics of a four-level system with two subsystems and our results conform to that of [1]. We see that the entropy has increased. This is due to the fact that each of the states of the subsystems has introduced a new degree of freedom, when separated, thereby increasing 
the disorder of the system. The numerical results also show that each of the thermodynamics does not depend on the sign of $\epsilon_{1}$. We also found that that each of the thermodynamics obeys all the existing laws guiding it even at extreme values of temperature. We also found that the four-level system is applicable to metals since it gives a linear relationship with temperature at very low temperatures. It gives the orbital electron contribution to the heat capacity.

Acknowledgement We wish to thank our anonymous referees for their positive and invaluable suggestions. Oladunjoye A. Awoga wishes to dedicate this work to his mother who died on October 08, 2011. She against all odds made sure I am educated and gave me hope in life. She inspired me to love academics. She sacrificed a lot for me. I am forever grateful to her.

Received 23 March 2012

\section{References}

[1] Awoga Oladunjoye A., Ikot Akpan N., Essiett Aniesua A. And Akpabio LOUIS E.: Thermodynamic properties of the harmonic oscillator and four level system. Appl. Phys. Rese. 3(2011)1.

[2] Kelly James J.: Physics 603: Methods of Statistical Physics. (2002), www.physics.umd.edu/courses/phys603/kelly/.

[3] Yoshioka D.: Statistical Physics. Springer, New York 2007.

[4] Nash Leonard K.: Elements of Statistical Thermodynamics. Addison-Wesley, Massachusetts 1974.

[5] Margenau H., Murphy G.M.: The Mathematics of Physics and Chemistry. Van Nostrand, New York 1947.

[6] Bence K.F., Hobson M.P. and Bence S.J.: Mathematical Methods for Physics and Engineering. Cambridge University Press, London 2002.

[7] Arfken G.: Mathematical Method for Physicists. Academic Press Inc, New York 1980.

[8] Ayres Frank, JR: Theory and Problems of Matrices. McGraw Hill, New York 1964.

[9] Cheng D., Qi H. And Xue A.: A survey on Semi-Tensor Product of Matrices. J. Sys. Sci. Complex. (2007), 304-322.

[10] Cheng D.: Semi-tensor product of matrices and its application to Morgen's problem. Sci. in China 44(2001), 3, 195-212.

[11] Steeb W.: Matrix Calculus and Kronecker Product with Applications and $C^{++}$ Programs. World Scientific, New York 1997. 
[12] Laub A.J.: Matrix Analysis for Scientists and Engineers. (2005) www.ecsecurehost.com/SIAM/ot91.html

[13] Sethna J.P.: Entropy, Order Parameters and Complexity. Cladevon Press, Oxford 2006, 157-163.

[14] Landau L.D. And Lifshitz E.M.: Statistical Physics. Pergamon Press, New York 1980, 195-198.

[15] Davydov A.S.: Quantum Mechanics. Pergamon Press, New York 1991, 41-46.

[16] Merzbacher E.: Quantum Mechanics. Wiley \& Sons Inc., New York 1976, 278291.

[17] Messiah A.: Quantum Mechanics. Willey \& Sons Inc., New York 1970, 331-338.

[18] Feynman R.P.: Statistical Mechanics. Addison-Wesley, New York 1972.

[19] Hanggi P. And Ingold G.: Quantum Brownian motion and the third law of thermodynamics. Acta Phys. Pol. B 37(2006), 5, 1537-1550.

[20] Kozliak E. And Lambert F.L.: Residual entropy. The third law and latent heat. Entropy 10(2008), 274-284. doi:10.3390/e10030270, http://dx.doi.org/10.3390/e10030270.

[21] Ingold G., Hanggi P. And Talkner P.: Specific heat anomalies of open quantum systems 2009 arxiv: quant-ph/0811.3509. doi:10.1103/physRevE.79.061105, http://dx.doi.org/10.1103/physRevE.79.061105.

[22] Hanggi P. And Ingold L.: Fundamental aspects of quantum Brownian motion. Chaos (2005), 1-12. doi:10.1063/1.1853631, http://dx.doi.org/10.1063/1.1853631.

[23] Ingold G., Lambert A. And Reynaud S.: Quantum dissipative Brownian motion and the Casimir effect. 2009 arxiv: quant-ph/0905.3608. doi:10.1103/PhysRevE.80.041113, http://dx.doi.org/10.1103/PhysRevE.80.041113.

[24] Hijar H. And de Zarate J.O.: Jarzynski equality illustrated by simple examples. Euro. J. Phys. 31(2010), 1097-1106.

[25] Tuckerman M.: Statistical Mechanics. (2006) www. Nyu.edu/classes/tuckerman/ /statmech/postscript/lecture4.pdf.

[26] Mabuchi H.: Lecture Notes on Advanced Quantum Mechanics. (2000) www.iks.cultechedu//hmabuchi.ph125.html.

[27] Andersen H.C.: Enthalpic- isobaric ensemble. J. Chem. Phys. 72(1980), 23842393.

[28] Wark K.: Thermodynamics. McGraw Hill, New York 1977.

[29] Kittel C.: Introduction to Solid State Physics, 6th edn. Willey \& Sons Inc., New York 1986, 100-140.

[30] Gradshteyn I.S. and Rhyzhik I.M.: Table of Integrals, Series and Product. Elsevier, Burlington 2007. 